involving twice-weekly sessions for 8 weeks. Patients were assessed pre- and post-programme by Chronic Respiratory Questionnaire (CRQ), the Hospital Anxiety and Depression Scale (HADS) and an incremental shuttle-walking test. Satisfaction was measured using the Client Satisfaction Questionnaire (CSQ). Results are expressed as mean (SD). Four patients aged 65-79 enrolled for this feasibility study, which was approved by the North of Scotland Research Ethics Service.

Results All patients completed the programme. The technology performed well: one patient missed a single session due to a temporary problem connecting to the Internet. Clinical improvements occurred in all patients, comparable to those in a conventional programme. Compared to pre-programme values, there were improvements in: shuttle walking, 88 (35) $\mathrm{m}$; CRO dyspnoea 8 (5.2); emotion 2.8 (1); fatigue 5.3 (3.7) and mastery 4 (2.6). HADS anxiety and depression scores reduced in three of four patients. Satisfaction scores were high: CSQ values 29-32.

Conclusion The system allowed delivery of effective group-based pulmonary rehabilitation to patients in their own homes and should improve accessibility for future patients. Larger studies are warranted.

\section{P52 IS A PULMONARY REHABILITATION (PR) PROGRAMME EFFECTIVE IN REDUCING SEVERE SYMPTOMS OF ANXIETY AND DEPRESSION IN PATIENTS WITH COPD?}

doi:10.1136/thx.2010.150979.3

S Harrison, N Greening, J Williams, S Singh. Pulmonary Rehabilitation Research Group, Glenfield Hospital, University Hospitals Leicester NHS Trust, Leicester, England

Introduction Patients with COPD have a high prevalence of anxiety and depression which has been associated with poorer health outcomes. PR has been found to be successful in reducing anxiety and depression. Previous studies have not differentiated between mild, moderate and severe symptoms of anxiety and depression and therefore the efficacy of PR are unproven in treating those across a spectrum of anxious and depressed symptoms. Our study aimed to explore the effectiveness of a PR programme in reducing mild, moderate and severe symptoms of anxiety and depression in patients with COPD.

Methods Patients with a clinical and spirometric (GOLD stage $\geq 2$ ) diagnosis of COPD were identified from the PR database in Leicester, UK. Patients were categorised into three groups based on their Hospital Anxiety and Depression Scale (HADS) scores pre PR ('none' 0-7, 'probable' 8-10 and 'presence' 11-14). These patients undertook an outpatient PR programme between 2000 and January 2009 at a single centre. Changes in HADS scores were retrospectively compared. The two subscales were analysed separately.

Results 518 patients were identified (mean (SD) age 69.2 (8.80) years $\mathrm{FEV}_{1} 39.9 \%$ (15.10) predicted 310 male). Patients with a 'probable' and a 'presence' of anxiety and depression had a reduction in HADS scores pre- to post- PR $(p<0.001)$. Patients who had 'none' did not have a reduction ( $p>0.05$ ) (Abstract P52 Figure 1). An ANOVA with post hoc analysis showed a difference in the amount of change pre to post PR between the three groups. Patients with a 'presence' had the greatest reductions in their scores $(\mathrm{p}<0.05)$

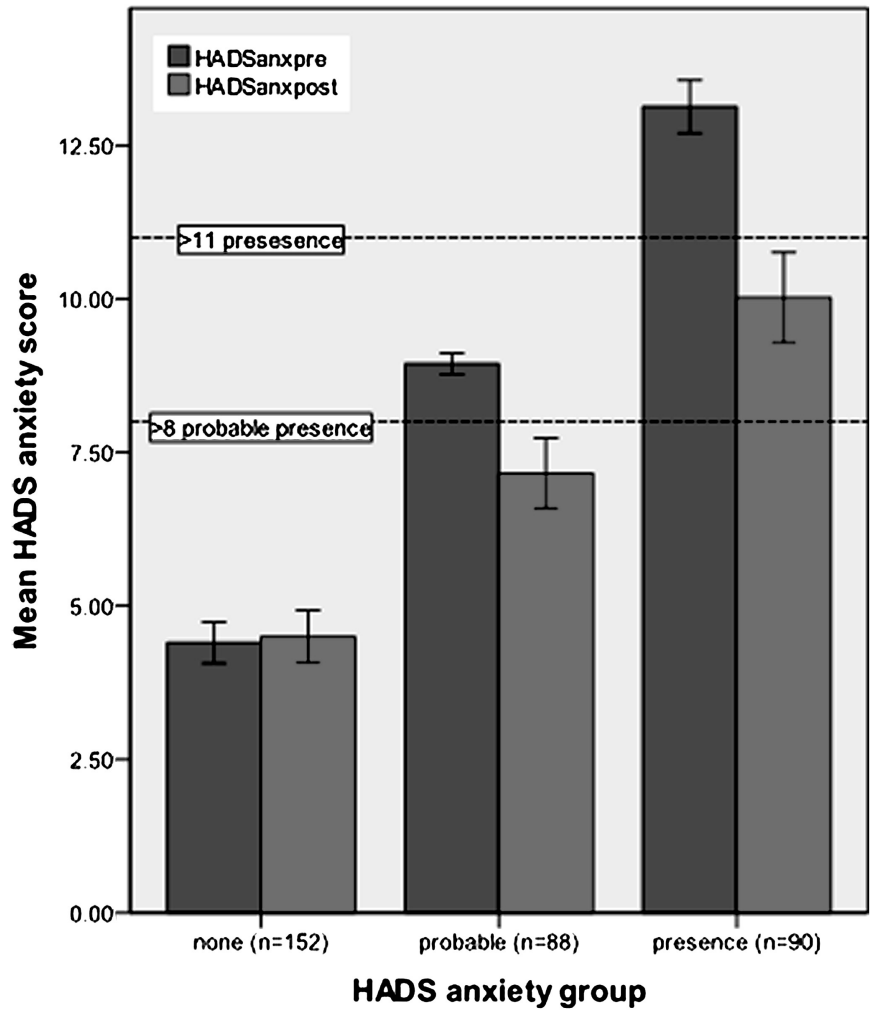

Error bars: $95 \% \mathrm{Cl}$

Abstract P52 Figure 1 Results from a paired t-test. Levels of anxiety pre and post PR as measured by the HADS.

Conclusions PR is effective in reducing moderate and severe symptoms of anxiety and depression in patients with COPD. However, $\mathrm{PR}$ is not effective in reducing severe symptoms to a level which represents no presence of anxiety and depression.

\section{P53 THE EFFECT OF CO-MORBIDITIES ON RESPONSE TO PULMONARY REHABILITATION}

doi:10.1136/thx.2010.150979.4

K A Ingram, R P Fowler, A L Clark, C M Nolan, W D Man. Harefield Pulmonary Rehabilitation Team and Biomedical Research Unit, Royal Brompton \& Harefield NHS Foundation Trust, Harefield, UK

Introduction Most patients with COPD are elderly, and the presence of co-morbidities is not uncommon. Co-morbidities are an important determinant of health related quality of life in patients with COPD. Although pulmonary rehabilitation (PR) is well known to improve exercise performance, health-related quality of life and symptoms, a proportion of patients are non-responders. We hypothesised that improvement in exercise capacity and healthrelated quality of life following PR may be limited in the presence of co-morbidities.

Methods A structured history was taken from 128 patients referred to an 8-week supervised outpatient PR programme. Individuals' selfreported co-morbidities were assessed by the Charlson index, which assigns to each disease a score that is proportional to the disease related risk of death. The calculated Charlson index did not include COPD in the individual's score as suggested in the original description. In 115 patients completing the programme, changes in incremental shuttle walk (ISW) and chronic respiratory disease questionnaire (CRQ) domains following PR were calculated, and 
compared between those patients with and without co-morbidities (Charlson index 1 or more vs 0 ) using unpaired t-test or non-parametric equivalent.

Results 84 patients (73\%) had a Charlson index of 1 or more. No difference in age, gender, FEV1, or MRC Dyspnoea score was seen between those with and without co-morbidities. Mean (SD) change in ISW following PR was not significantly different between those with and without co-morbidities (63 (96) vs 33 (88); $p=0.13$ ). Similarly there were no significant differences in CRQ-D change (4.1 (6.4) vs 5.7 (7.1); $\mathrm{p}=0.26$ ), CRQ-F change (2.6 (5.1) vs 4.4 (5.7); $\mathrm{p}=0.13)$, CRQ-E change (3.6 (7.8) vs $6.4(7.7) ; \mathrm{p}=0.09)$ or CRQ-M change $(2.7$ (4.8) vs $2.3(6.9) ; p=0.75)$. No association was seen between Charlson index and change in ISW following PR (Spearman rank=0.09; $\mathrm{p}=0.32$ ).

Discussion The prevalence of co-morbidities in COPD patients undergoing PR is high. The presence of co-morbidities does not seem to affect patients' response to pulmonary rehabilitation.

\section{Changing patterns of mycobacterial disease P54 DO THE NICE NEW ENTRANT TB SCREENING GUIDELINES
UNDER-DIAGNOSE CASES OF LATENT TB INFECTION?}

doi:10.1136/thx.2010.150979.5

D Thomas, M Jarvis, A Williams. The Royal Bournemouth and Christchurch Hospitals NHS Foundation Trust, Bournemouth, UK

Introduction In 2006, $72 \%$ of active TB cases in the UK occurred in people born overseas (HPA 2008). 48\% of new entrants with TB were diagnosed within 5 years of entering the UK and 19\% within 2 years (HPA 2008). It is a priority therefore, to identify and appropriately treat those infected with latent $\mathrm{TB}$ infection through TB screening programmes (DH 2004). NICE (2006) TB new entrant screening guidelines allow certain groups of new entrants to be screened solely via chest $\mathrm{x}$-ray (CXR), omitting a Tuberculin Skin Test (TST). This potentially under-diagnoses latent TB Infection (LTBI). The aim of this study was to determine whether NICE (2006) criteria are adequate in detecting latent TB.

Method A retrospective case-note analysis of new entrants over a 44month period (2006-2009). All patients were screened using a locally developed 'Dorset' algorithm that combined CXR and TST unless contraindicated (see Abstract P54 Figure 1). Each case was then re-evaluated using the NICE algorithm. This allowed direct comparison of each algorithm to detect LTBI.

Results 547 new entrants were referred locally for TB screening (2006-2009). 397 attended. 41 (10.3\%) patients (all HIV-ve) were diagnosed with LTBI, based on the following outcomes:

- Abnormal CXR and strongly positive TST=14 (34\%).

- Normal CXR but strongly positive TST=18 (44\%).

- Abnormal CXR but normal TST=9 (22\%).

Comparison of the two algorithms showed that while all 41 cases were detected using the Dorset algorithm, only 27 cases (65.8\%) were detected using the NICE algorithm. This represents a $34.1 \%$ shortfall in LTBI detection using NICE (95\% CI 19.63\% to $48.67 \%$, $99 \%$ CI $15.04 \%$ to $53.26 \%$ ).

Discussion This study demonstrated that through the omission of TST, the NICE algorithm missed 14 (34.1\%) cases of LTBI compared with the Dorset algorithm. While alternative screening methods such as IGRA are increasing in recognition, these continue to be an expensive option if not provided locally. Therefore TB services without routine access to IGRA can significantly improve their detection of latent TB by simply combining their existing screening tools.

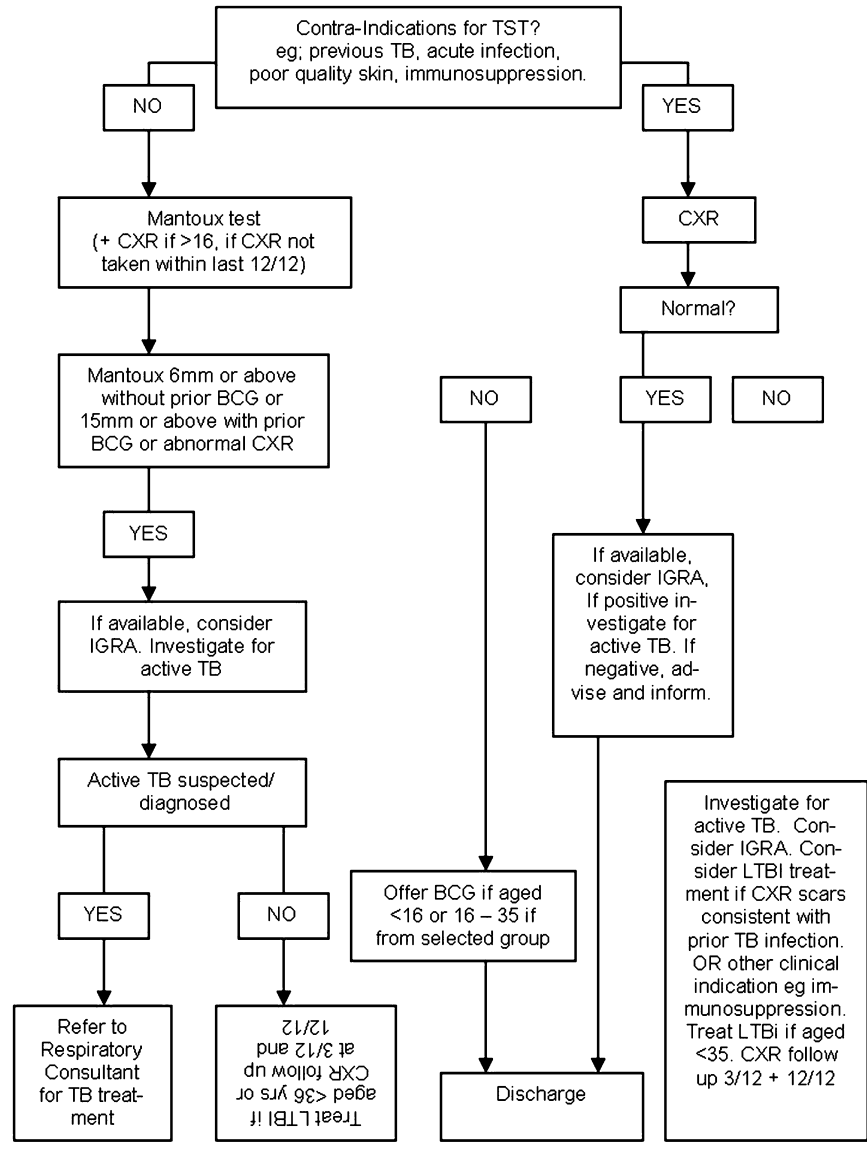

'Inform and advise' information should be offered to all New Entrants.

Treatment for LTBI should be offered to those aged $<35$ and any age of health care worker or HIV +ve individual.

Selected group - from sub-Saharan Africa or a country with TB incidence $>500 / 100,000$

Abstract P54 Figure 1 Dorset new entrant TB screening algorithm (2009).

\section{P55 POST-BRONCHOSCOPY SPUTUM: INCREASING THE DIAGNOSTIC YIELD IN SMEAR NEGATIVE PULMONARY TUBERCULOSIS}

doi:10.1136/thx.2010.150979.6

${ }^{1} \mathrm{P}$ M George, ${ }^{1} \mathrm{M}$ Mehta, ${ }^{1} \mathrm{~J}$ Dhariwal, ${ }^{1} \mathrm{~A}$ Singanayagam, ${ }^{1} \mathrm{C}$ E Raphael, ${ }^{1} \mathrm{M}$ Salmasi, ${ }^{1} \mathrm{D}$ W Connell, ${ }^{1} \mathrm{P}$ Molyneaux, ${ }^{1} \mathrm{M}$ Wickremasinghe, ${ }^{2} \mathrm{~A}$ Jepson, ${ }^{1} \mathrm{O} \mathrm{M}$ Kon. ${ }^{1}$ Chest and Allergy Department, St Mary's Hospital, Imperial College Healthcare NHS Trust, London, UK; ${ }^{2}$ Department of Microbiology, St Mary's Hospital, Imperial College Healthcare NHS Trust, London, UK

Background The prevalence of smear negative pulmonary tuberculosis (PTB) is increasing. At many centers, active PTB suspects who are Acid-Fast Bacilli (AFB) smear negative or non-productive of sputum undergo fiber optic bronchoscopy for bronchoalveolar lavage but post bronchoscopy sputum (PBS) sampling is not routine. The aim of the study was to establish the clinical utility of PBS sampling in this subgroup of patients with active PTB.

Methods A retrospective study of all patients attending a central London University hospital with microbiologically confirmed PTB between January 2004 and December 2009. Patients who were AFB smear negative or non-productive of sputum were eligible for the study if a sputum sample was obtained within 7 days of bronchoscopy.

Results The cohort $(\mathrm{n}=50)$ was heterogeneous - 29 were male (58\%), 12 were infected with HIV (24\%), 19 were of African origin (38\%), 17 were white Caucasian $(34 \%)$ and four were from the 